\title{
Sensing of organic substances by controlling surface polarization of electrodes
}

\author{
Member Kenshi Hayashi (Kagoshima University) \\ Non-member Kazuaki Moriyama (Kagoshima University)
}

Photovoltaic effects of semiconductor were examined for chemical sensing. Light induced potential change influences surface electrical polarity through lighy generated carriers, then characteristics of electrode changes and the electrode respond to various chemicals. Odor and taste substances have various electrical property and affect the light induced potential change. We can detect very sensitively electrolyte or nonelectrolyte, such as sucrose, ethanol and mono sodium glutamate by changes of photo voltage. The results suggest that characteristics of the transducer can be controlled and information of chemicals multiplexed in a signal from a single transducer.

keywords: sensor, chemical senses, taste, odor, surface polarization

\section{INTRODUCTION}

Detecting taste and odor substances is difficult because there exist numerous chemicals and the sensor must be able to respond to such various chemicals, those expand electrolytes such as salt or acid to various nonelectrolytes. On the other hand, high sensitivities are required for taste and odor sensors. Furthermore, artificial sensors must recognize quality and quantity of chemicals in the same manner as the senses of gustatory and olfactory.

In the present, information of taste and odor cannot be treated quantitatively, and methods to transform taste and odor information into data for communication are not realized. Some attempts were done to realize taste or odor sensing[1,2]; the multichannel lipid membrane sensor or the multichannel quartz resonance sensor, but the sensitivity and quality recognizing are inferior to biological chemical senses.

We examined a photovoltaic effect of semiconductors; photo voltage affected in an organic semiconductor[3] and p-Si $[4,5]$. In the present paper, high sensitive chemical detection was achieved using $\mathrm{p}$-Si by means of precise light intensity control. To sense taste or odor, it is indispensable to detect various substances, such as electrolyte and nonelectrolyte. The surface of a semiconductor changes its electrical property by irradiating light through light generated carriers. The present sensor controls its surface electrical polarization by means of irradiating light intensity. The changes of polarization affect characteristics of interaction between the surface of electrode and detecting chemicals, i.e., negatively and positively charged surfaces can detect cations and anions in a solution, respectively. Thus the present method has an ability to detect quality of taste or odor, which depends mainly on electrical property and structure of chemicals. Furthermore, a neutral surface interacts with nonelectrolyte in solution with hydrophobic effects of chemicals in water[6,7]. Consequently a surface polarization is modified from the neutral state by light irradiation, then a detailed information about chemicals will be obtained. The present method also has ability to detect various chemicals with high sensitivity. The photovoltaic effect is influenced by surface condition of the electrode; the light induced potential is easily modified by ionic composition and chemical adsorption of detecting substances, because the photo voltage occurs at the just boundary surface between solution and semiconductor.

\section{MATERIALS AND METHODS}

\subsection{Electrode and Cell}

P-type silicon wafer $0.38 \mathrm{~mm}$ in thickness and $7.5 \mathrm{ohm}-\mathrm{cm}$ in resistivity was used. Lead wire was attached by the use of a carbon paste on nickel plated on backside of the $\mathrm{Si}$ wafer. Mirror polished surface of the wafer was touched to the solution in the cell. Then electric potential between the $\mathrm{Si}$ electrode and a reference electrode, $\mathrm{Ag} / \mathrm{AgCl}$ enclosed in $100 \mathrm{mM} \mathrm{KCl}$, was measured through a high input

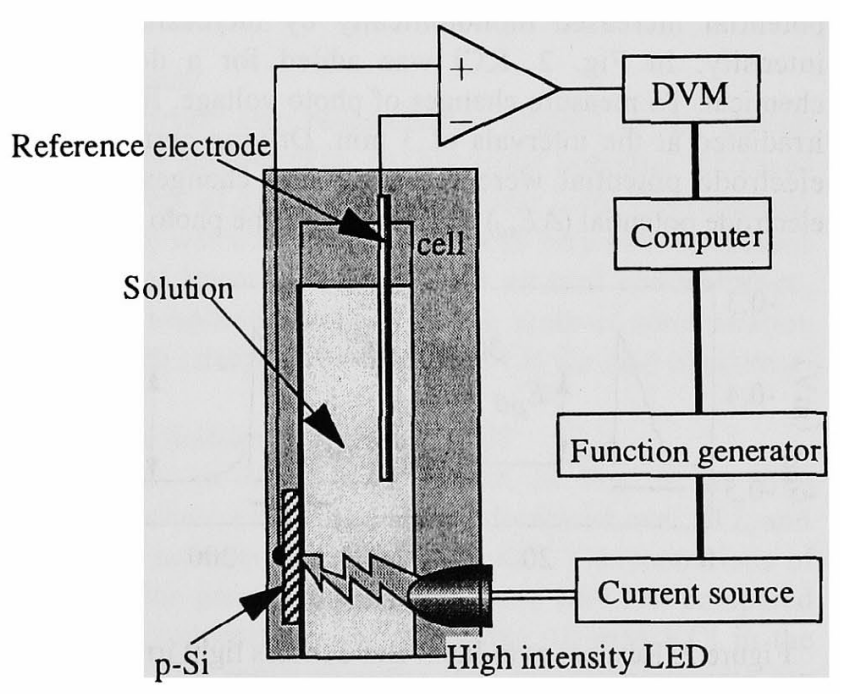

Figure 1. Experimental setup 
impedance amplifier and a digital voltmeter. The experimental setup is shown in Fig. 1.

The electrode was kept in alkaline solution, $\mathrm{pH} 9.18$ standard buffer solution, to stabilize a surface condition. Before experiments, the electrode was dipped in $10 \mathrm{mM}$ $\mathrm{KCl}$, which was standard experimental solution, and kept one hour to get stable electrode potential and photo voltages. A stability of the static potential was a few $\mathrm{mV}$ in one hour.

\subsection{Light Irradiation}

A source of light was a $150 \mathrm{~W}$ halogen lamp or a high intensity LED (light emitting diode). In the case of the halogen lamp, an intensityof irradiating light was controlled manually by changing an electric potential of regulated power supply for the bulb, and light intensities were designated in an arbitrary unit. Light intensity of LED was controlled by driving current. Light intensity $\left(L_{i}\right)$ of LED was denoted by

$$
L_{I}=10 \log (I+1) / \log \left(I_{a}+1\right)
$$

where $I$ is driving current of LED and $I_{a}$ is maximum driving current. The current was measured in $\mathrm{mA}$ unit and $I_{a}$ was set to $10 \mathrm{~mA}$. The light intensity was controlled through a function generator, which generates temporally changing exponential waveform for output voltage to control current source. The waveform of driving current was adopted to obtain linearly-changing photo voltage in time course.

\subsection{Measurement of Light Induced Potential Changes}

Light was irradiated from the backside of the Si electrode in the case of the halogen lamp through a light guide for experiments described in section 3.1. Light from LED was irradiated from the front of the cell (Fig. 1).

Time courses of the light induced potential changes of electrode $\left(E_{e d}\right)$ was shown in Fig. 2. The electrode potential increased monotonically by increasing light intensity. In Fig. 2, $\mathrm{KCl}$ was added for a detecting chemical. To measure changes of photo voltage, light was irradiated at the intervals of $3 \mathrm{~min}$. Driving current and electrode potential were recorded, and changes of the electrode potential $\left(\Delta E_{e d}\right)$ and changes of the photo voltage

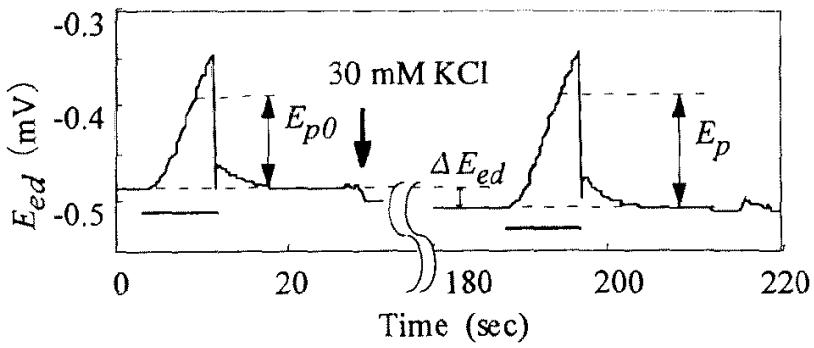

Figure 2. Responses to light. Bar denotes light irradition. $\Delta E_{p}=E_{p}-E_{p 0}$ in each light intensity, where $E_{p 0}$ is photovoltage in $10 \mathrm{mM} \mathrm{KCl}$.
$\left(\Delta E_{p}\right)$ were calculated for each $L_{I}$ in Eq. (1). Here, $\Delta E_{e d}$ and $\Delta E_{p}$ are changes of $E_{e d}$ (electrode potential) and $E_{p}$ (photo voltage) from the case of $10 \mathrm{mM} \mathrm{KCl}$ solution (see Fig. 2).

\section{RESULTS}

\subsection{Effect of $\mathrm{pH}$ of Solution}

To decide surface electric polarities of the p-Si electrode, responses to $\mathrm{KCl}$ were measured. In the case of neutral $\mathrm{pH}$ condition, the potential changed negatively for low light intensity and positively for high light intensity. The results mean that the electrode surface is positive in dark and negative in high light intensity condition and electrically neutral surface was obtained in medium light intensity. The value for the neutral surface had been unstable if no alkaline preconditioning, described in 2.1, was carried out.

In the case of alkaline solution, $\Delta E_{e d}$ changed positively in dark condition, then the surface of the electrode was negatively charged by $\mathrm{OH}^{-}$in the solution. The precondition of the electrode in alkaline solution makes initial surface electric charge stable in the present electrode.

Nonelectrolyte can be detected when the surface is

(a) in neutral $\mathrm{pH}$ condition

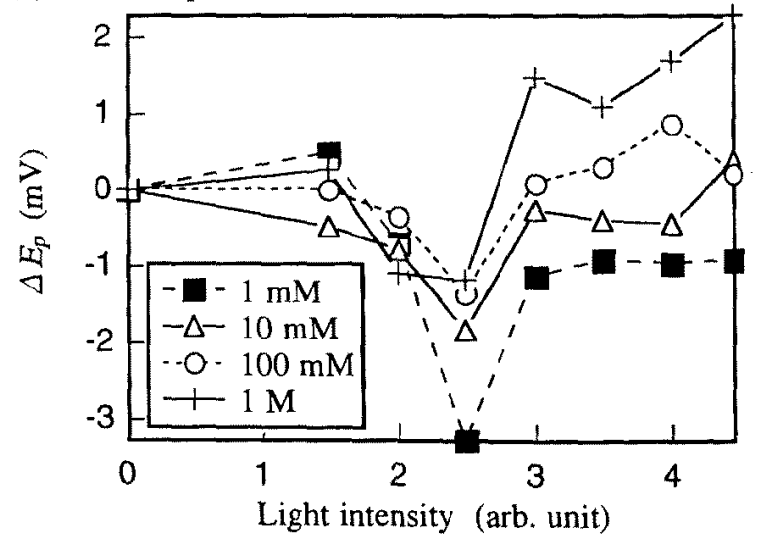

(b) in alkaline condition

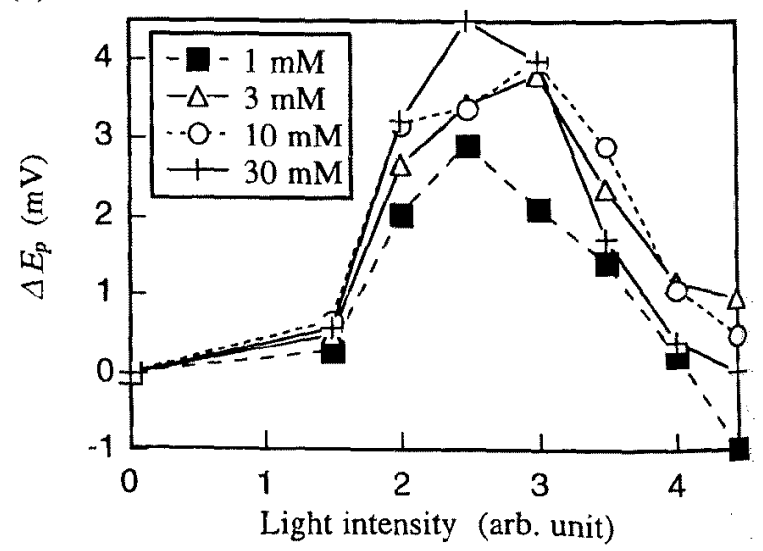

Figure 3. Responses to sucrose. Neutral $\mathrm{pH}$ and alkaline solution were used for diluting solution. 


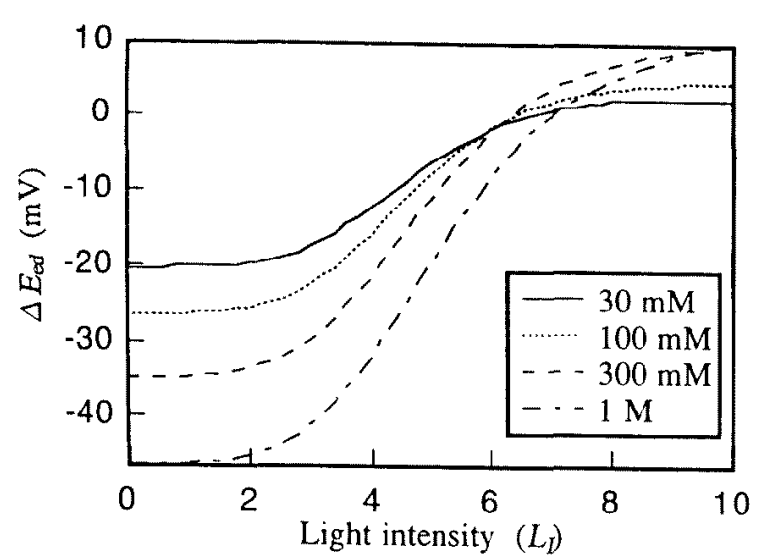

Figure 4. Responses to $\mathrm{KCl}$.

neutral. However, responses to nonelectrolyte at medium light intensity were also achieved in alkaline solution. Figure 3 represents responses to sucrose. Peaks of responses to sucrose appeared near 2.5 in both cases, although the directions of peak were opposite. The present results suggest that the neutral surface was realized in alkaline solution and neutral $\mathrm{pH}$ solution. Although the detailed mechanism is not clear at the present, positively charged inner boundary inside the Si surface is formed and that the positive boundary should be neutralized by light generated carriers.

The photo responses did not appear in acid solution. It can be explained by extinction of potential decay, which is formed by space charge layer, in Si near the surface by adsorption of $\mathrm{H}^{+}$.

\subsection{Responses to Chemicals}

\section{(i) $\mathrm{KCl}$}

Responses to $\mathrm{KCl}$ were measure using the LED light source. LED was used to improve resolution of responses. Responses to $\mathrm{KCl}$ also decide the polarity of surface electric charge in each light intensity. Figure 4 represents the $\mathrm{KCl}$ responses of electrode potential $\Delta E_{e d}$. Neutral surface was achieved at $L_{\Gamma}=6$, where photo voltage was $c a .40 \mathrm{mV}$. The value was the similar to the case of backside irradiation; the photo voltage was also ca. $40 \mathrm{mV}$ at 2.5 of light intensity scale shown in Fig.3. $\Delta E_{p}$ changed monotonically by light intensity increment. The increase was due to surface potential change, i.e., difference between flat band electric potential and static electric potential, which related to $E_{e d}$ in dark, increased by increment of $\mathrm{KCl}$ concentration.

\section{(ii) Sucrose}

Responses to sucrose were shown in Fig. 5. Electrode potential slightly changed by addition of sucrose. $\Delta E_{p}$ also changed and maximum responses were attained at $L_{F}=6$ where the no response to $\mathrm{KCl}$ was appeared, i.e., at neutral surface. In lower concentration, the electrode can detect sucrose at $0.1 \mu \mathrm{M}$, then the present electrode has very high sensitivity compared with biological taste sensation, where sucrose can be detected ca. $10 \mu \mathrm{M}$. The reason why the (a) High concentration

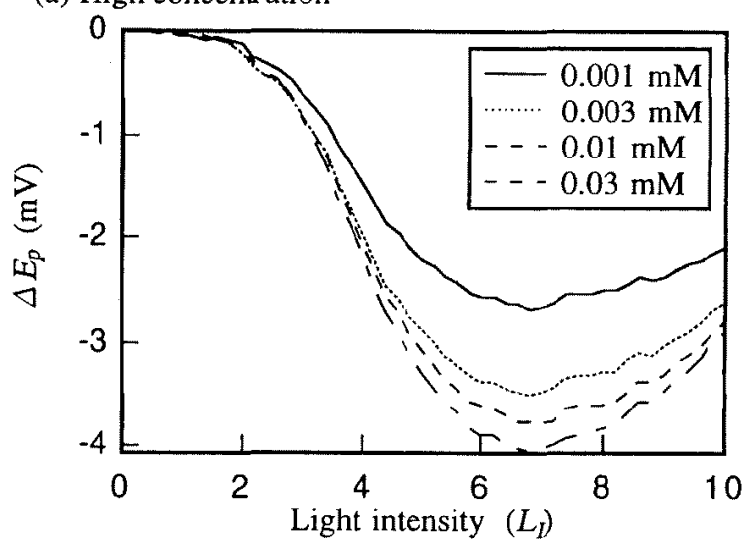

(b) Low concentration

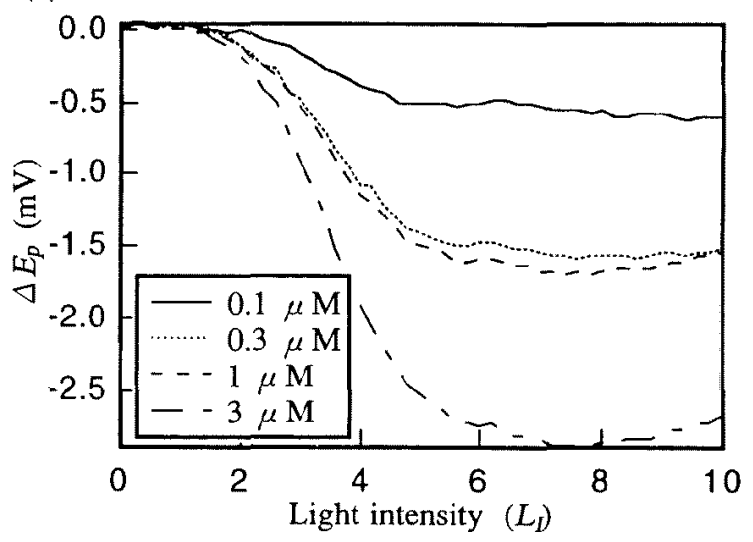

Figure 5. Responses of photo voltage to sucrose.

$\Delta E_{p}$ was negative and opposite to the case of $\mathrm{KCl}$ is supposed that $\Delta E_{e d}$ changed positively and opposite to the $\mathrm{KCl}$ case. Nevertheless, it is to be noted that sucrose response patterns, those had peak, made by light intensity changes were different from monotonic patterns of $\mathrm{KCl}$. Therefore, electrolytic $\mathrm{KCl}$ and nonelectrolytic sucrose will be distinguished easily by the use of response patterns.

\section{(iii) Ethanol}

The electrode also responded to ethanol in the similar manner to sucrose (Fig. 6). Electrode potential increased and the peak of $\Delta E_{p}$ also appeared near $L_{\Gamma}=6$. The threshold to ethanol was $0.02 \%$; the value was very low. Responses to ethanol linearly changed with ethanol concentration, whereas responses changed by log scale of concentration and almost saturated above $0.01 \mathrm{mM}$ in the case of sucrose.

\section{(iv) Mono Sodium Glutamate (MSG)}

Responses to MSG were shown in Fig. 7. In high concentrations, electrode potential decreased over all $L_{I}$ and response patterns were similar to $\mathrm{KCl}$. Concentrations of MSG in the present experiments were very low compared with the concentration of coexisting $10 \mathrm{mM} \mathrm{KCl}$ in the solution. The result suggests that anionic glutamate ion adsorbed to the surface of the electrode. In lower concentration, electrode potential changed similar fashion 
(a) High concentration

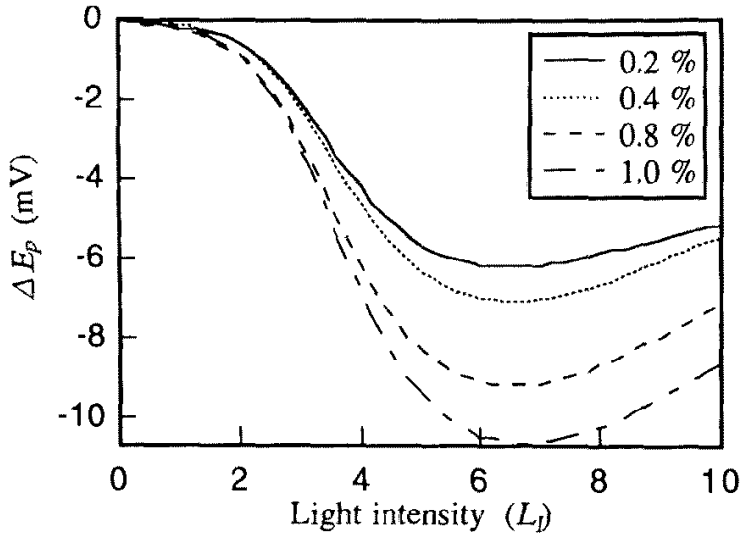

(b) Low concentration

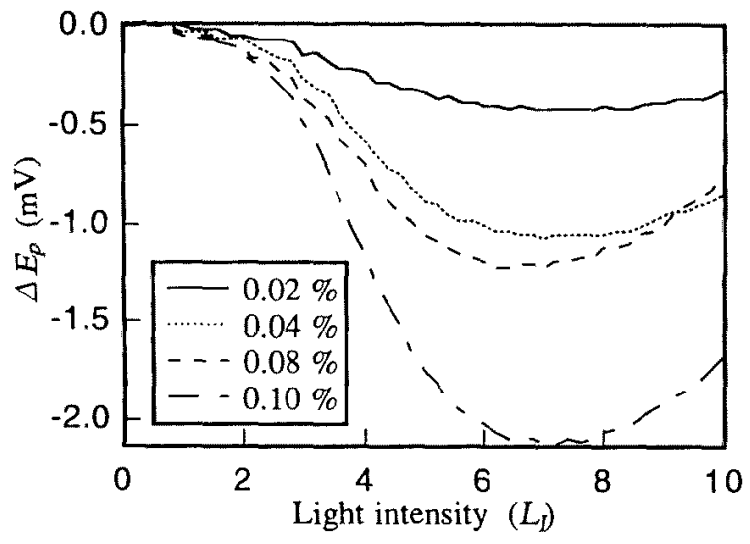

Figure 6. Responses of photo voltage to ethanol.

with sucrose or ethanol. $\Delta E_{p}$ also had the same pattern with $\mathrm{KCl}$ in high concentration, and with ethanol and sucrose in lower concentration. It is the future task to decompose the present pattern to each pattern of $\mathrm{Na}^{+}$and glutamate.

\section{DISCUSSION}

The present method had high sensitivities to nonelectrolytes, those were difficult to detect electrically. For example, sucrose can be detected at concentration of a few ten ppb order. Although the present experiments were carried out to detect chemicals in water solution, the present results suggest that the electrode can be utilized for odor sensing.

Scatters of responses were ca. $0.5 \mathrm{mV}$ in both halogen lamp and LED. On the other hand, peak position of response in light intensity had high repeatability. A static potential for a silicon electrode with no preconditions was unstable, then the precondition in alkaline solution was importance to get high repeatability of chemical responses, because the value of the static potential and its temporal stability influence the magnitude of responses to chemicals.

The present electrode and experimental method have some advantages for chemical sensing, i.e., 1) the transducer, $\mathrm{p}$-Si, can be polished after experiment then few worse occurs in electrode property, 2) photo responses can

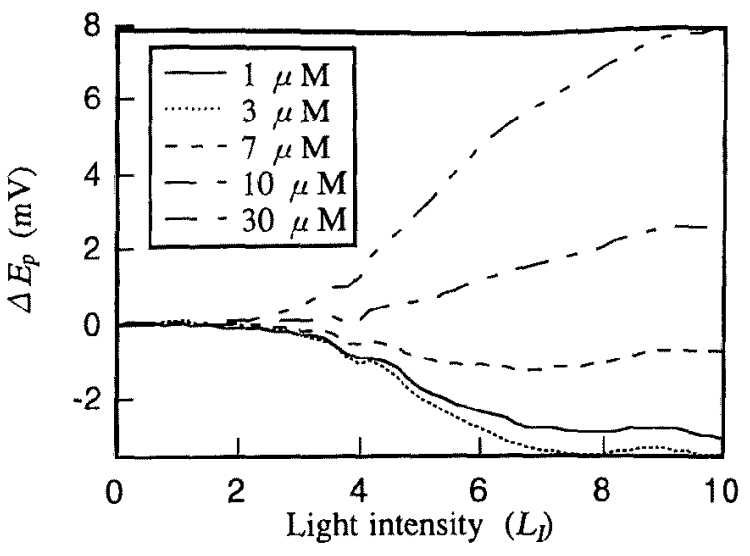

Figure 7. Responses of photo voltage to MSG.

be measured by use of non electrochemical reference electrodes, because it requires only difference between dark and light condition in each chemical concentration.

The present method can obtain information about chemical substances with one electrode. Anions can be detected with the electrode irradiated with low light intensity and cations with the electrode under high light intensity. Nonelectrolyte or neutral parts of chemicals will interact with the electrode surface in neutral electric charge density. In the present study, light intensity was changed continuously to detect the response patterns to chemicals, but sucrose and ethanol cannot be discriminated with the patterns. For electrolytes, responses appeared with no peak, then the electrode has an ability to distinguish electrolytes and nonelectrolytes, and to decompose elements of the mixture solution. The results denote that the present electrode can be used for chemical sensor for taste or odor sensing.

(Manuscript received 29 Mar. 1996, revised 15 Aug. 1996)

\section{ACKNOWLEDGMENT}

Part of the present study was supported by the frontier research in telecommunications.

\section{REFERENCES}

[1] K. Hayashi, M. Yamanaka, K. Toko and K. Yamafuji, "Multichannel taste sensor using lipid membranes", Sens. Actuators, B2, pp.205-213, 1990.

[2] T. Nakamoto, K. Fukunishi and T. Moriizumi, "identification capability of odor sensor using quartz resonator array and neutral network pattern recognition", Sens. Actuators, B1, pp.473-476, 1990.

[3] K. Hayashi, "Sensing of chemical substances using light induced potential changes of organic membranes", Sens. Materials, 7, pp.13-22, 1995.

[4] K. Hayashi, K. Moriyama, "Chemical sensing and response ability control using photovoltaic effect of semiconductor", Tech. Dig. 13th Sens. Symp., pp.241-244, 1995.

[5] M. Shimizu, Y. Kanai, H. Uchida and T. Katsube: 
Sens. Actuators, B20 p.119, 1994.

[6] C. Tanford, The Hydrophobic Effect, WileyInterscience, 1980.

[7] J. N. Israelachivili, Intermolecular and Surface Forces, Academic Press, 1985.
Kenshi Hayashi (Member) He was born in 1960. He received the B. E. degree in 1984, and the M. E. degree in 1986 and the Dr. Eng. Degree in 1990 from Kyushu University. From 1990, he was a Research Associate in Department of Electronics of Faculty of Engineering, Kyushu University. From 1992, he was an associate Professor in Department of Electrical and Electronic Engineering, Faculty of Engineering, Kagoshima University. His main research interests is bio electronics.

Kazuaki Moriyama (Non-member) He was born in 1970. He received the $B$. E degree in 1994, and the M. E. degree in 1996 from Kagoshima University. He now works at Kinden Inc. 